\title{
Keratin 80 promotes migration and invasion of colorectal carcinoma by interacting with PRKDC via activating the AKT pathway
}

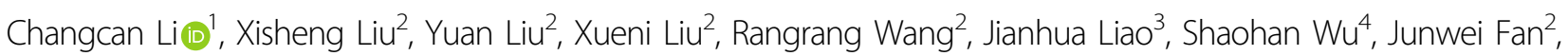
Zhihai Peng ${ }^{2}$, Bin $\mathrm{Li}^{5}$ and Zhaowen Wang ${ }^{2}$

\begin{abstract}
Little is known about the function of Keratin 80 (KRT80), an epithelial keratin, in cancer. This study investigated the role of KRT80 in the prognosis of colorectal carcinoma (CRC) and the underlying mechanisms involved in CRC migration and invasion. We analyzed the expression of KRT80 using The Cancer Genome Atlas and Oncomine databases. Higher expression of KRT80 was found to be significantly associated with multiple pathological parameters, lower disease-free survival, and overall survival in CRC patients. Also, KRT80 was an independent prognostic indicator for CRC.

Furthermore, altered KRT80 expression impacted migration and invasion of CRC cells, as well as the expression of epithelial-mesenchymal transition (EMT)-related markers and cell morphology via the AKT pathway. Inhibiting the expression of AKT could reverse these phenomena. Liquid Chromatograph Mass Spectrometer/Mass Spectromete, Coimmunoprecipitation, and laser scanning confocal microscopy techniques showed that KRT80 could interact with protein kinase, DNA-activated, catalytic polypeptide (PRKDC). Suppressing PRKDC could inhibit the expression of AKT and EMT, as well as the migration and invasion of CRC cells. Taken together, these results demonstrated that KRT80 was an independent prognostic biomarker for CRC and promoted CRC migration and invasion by interacting with PRKDC via activation of the AKT pathway.
\end{abstract}

\section{Introduction}

Although prominent advances have been made in the treatment of colorectal carcinoma (CRC), it remains the most common gastrointestinal cancer worldwide, with high morbidity and mortality rates ${ }^{1}$. Around $25 \%$ of newly diagnosed patients with CRC have metastasis, whereas $50 \%$ of patients with localized tumors will eventually develop metastasis, with most of these tumors being

\footnotetext{
Correspondence: Zhaowen Wang (zhaowenwangzhww@163.com)

${ }^{1}$ Department of General Surgery, Tongji Hospital, Tongji University School of Medicine, Shanghai 200065, China

${ }^{2}$ Department of General Surgery, Shanghai General Hospital, Shanghai Jiao Tong University School of Medicine, Shanghai 200080, China

Full list of author information is available at the end of the article.

These authors contributed equally: Changcan Li, Xisheng Liu, Yuan Liu

Edited by A. Stephanou
}

unresectable $^{2}$. The available clinical biomarkers have unsatisfactory sensitivity and specificity for CRC prognostic evaluation ${ }^{3}$. Identifying specific altered genes and/or biomarkers with high sensitivity and specificity will be invaluable for early diagnosis and prognosis of CRC.

Keratins are intermediate filament cytoskeletal proteins of epithelial cells that are responsible for their structural integrity ${ }^{4}$. Keratins can be divided into two types: acidic or type I and basic or neural type II. Keratin 80 (KRT80) belongs to type II, along with Keratin 7 (KRT7), Keratin 8 (KRT8), and Keratin 78 (KRT78). KRT80 gene is located on chromosome 12q13 and encodes a 452-amino-acid protein with a calculated molecular mass of $50.5 \mathrm{kDa}^{5,6}$. 

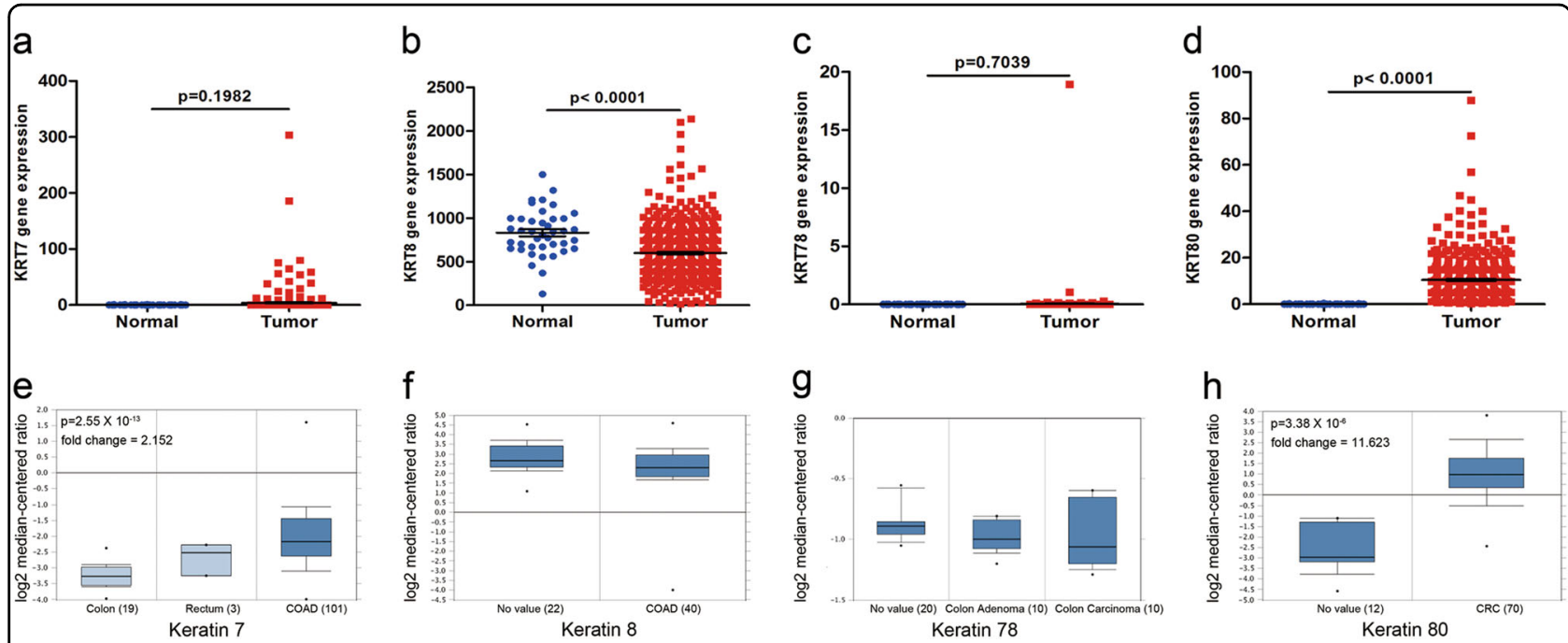

Fig. 1 Keratin family type II RNA expression on The Cancer Genome Atlas (TCGA) and the Oncomine databases a-d TCGA showed expression of keratin 7 (KRT7), keratin 8 (KRT8), keratin 78 (KRT78), and keratin 80 (KRT80) in normal mucosa and colon adenocarcinoma (COAD). e KRT7 expression in colorectum (TCGA Colorectal). $\mathbf{f ~ K R T 8 ~ e x p r e s s i o n ~ i n ~ c o l o n ~ ( A l o n ~ C o l o n ) . ~ g ~ K R T 7 8 ~ e x p r e s s i o n ~ i n ~ c o l o n ~ ( S k r z y p c z a k ~ C o l o r e c t a l ~ 2 ) . ~}$ h KRT80 expression in colorectum (Skrzypczak Colorectal 2)

Keratins are expressed in many tumors including carcinomas, sarcomas, and trophoblastic neoplasms ${ }^{7}$. Keratins are tissue-specific and expressed in a differentiationdependent manner. They are typical markers for epithelial cells ${ }^{8}$. Several studies have reported that keratins are extensively expressed in many malignant epithelial cells and play an important role in the regulation of cell migration and invasion ${ }^{9,10}$. However, the correlation between KRT80 expression and cancer has not been reported, not to mention in CRC. Hence, we focused on the role of KRT80 in CRC.

In this study, the clinical significance and related mechanisms of KRT80 in CRC were investigated. The Cancer Genome Atlas (TCGA) and Oncomine databases were used to predict the expression of KRT80 in CRC. High expression of KRT80 was demonstrated to be an independent prognostic indicator for CRC. Next, in vitro experiments showed that KRT80 was related to migration and invasion of CRC cells via the AKT pathway, the expression of epithelial-mesenchymal transition (EMT) markers and change of CRC cell morphology. Using Liquid Chromatograph Mass Spectrometer/Mass Spectrometer (LC-MS/MS), Co-immunoprecipitation (Co-IP), and laser scanning confocal microscopy (LSCM), we demonstrated that KRT80 interacted with Protein Kinase, DNA-Activated, Catalytic Polypeptide (PRKDC), also called as DNA-Dependent Protein Kinase Catalytic Subunit in CRC. Suppression of PRKDC could inhibit the AKT pathway, EMT, and the migration and invasion of CRC cells. These findings showed that KRT80 may play a vital role in CRC.

\section{Results}

\section{KRT80 is upregulated in human CRC tissues}

The keratin family type II RNA expression was examined in the TCGA and Oncomine databases. Only KRT80 showed significant changes in CRC tissues, as compared with normal adjacent mucosa (Fig. 1d, h). KRT7, KRT8, and KRT78 had no significant changes (Fig. 1a-c, e-g).

Next, 40 paired CRC samples were assessed for KRT80 mRNA expression by quantitative real-time PCR (qRT$\mathrm{PCR})$. The relative expression $(\Delta \mathrm{Ct})$ of KRT80 in cancerous tissues was $10.56 \pm 1.60$, and $13.07 \pm 2.29$ in adjacent normal tissues $(p<0.001)$. A total of 33 tumor samples (82.5\%) showed higher KRT80 expression in CRC tissues as compared with paired normal mucosa, with 28 tumor samples $(70 \%)$ having at least a twofold increase (Fig. 2a). Western blot analysis confirmed that the protein level of KRT80 was significantly upregulated in CRC tissue samples as compared with matched normal tissues (Fig. 2b). These results demonstrated that KRT80 expression was increased at the transcriptional and translational levels in CRC tissues.

\section{Association between KRT80 expression and clinicopathological parameters for CRC}

To determine the association of clinicopathological characteristics with KRT80 expression, immunohistochemistry (IHC) analysis was performed using a tissue microarray (TMA) containing 120 primary CRC samples and paired adjacent normal mucosae. KRT80 localized in the cytoplasm, and significant differences were observed 


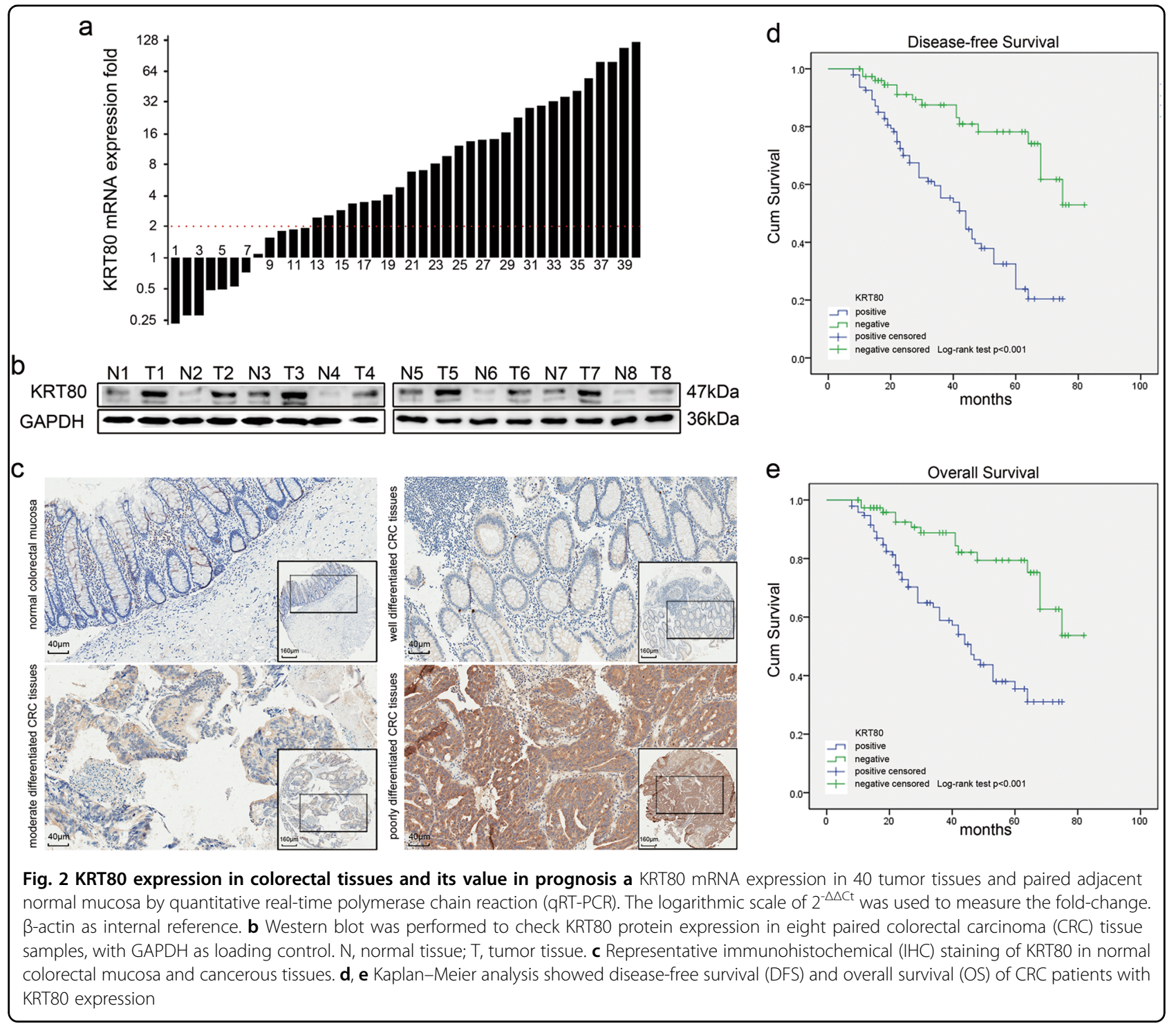

Table 1 Expression of KRT80 protein in paired normal tissues and CRC tissues

\begin{tabular}{lllll}
\hline Tissue sample & $\boldsymbol{n}$ & \multicolumn{2}{l}{ KRT80 expression } & \multirow{2}{*}{$\boldsymbol{P}$ value } \\
\cline { 3 - 4 } & & Negative (\%) & Positive (\%) & \\
\hline Normal tissue & 120 & $65(54.2)$ & $48(45.8)$ & $0.01^{\mathrm{a}}$ \\
Cancerous tissue & 120 & $48(40.0)$ & $72(60.0)$ & \\
\hline
\end{tabular}

${ }^{\text {a }}$ Implies statistical difference, $P$ value is based on the chi-square test

in KRT80 expression between normal mucosa and CRC tissues (Fig. 2c). KRT80 protein staining was higher in the primary cancer samples as compared with adjacent normal mucosa (Table 1). Also, KRT80 staining increased with the increase in tumor grade. Elevated KRT80 expression was significantly associated with several clinicopathological factors, including $\mathrm{T}$ stage, $\mathrm{N}$ stage, $\mathrm{M}$ stage, the American Joint Committee on Cancer (AJCC) stage, differentiation, and vessel invasion (Table 2).

\section{Survival analysis and prognostic significance of KRT80 expression in CRC}

To assess the association between KRT80 expression and survival of CRC patients, Kaplan-Meier curves with log-rank test was used to determine disease-free survival (DFS) and overall survival (OS) in 120 CRC patients. The group with high levels of KRT80 expression in tumors had poorer DFS and OS as compared with the group with lower KRT80 expression (Fig. 2d, e).

In addition, Cox proportional hazards model was used for univariate and multivariate analyses of DFS and OS. In the univariate analysis, $\mathrm{T}$ stage, $\mathrm{N}$ stage, $\mathrm{M}$ stage, AJCC stage, differentiation, vessel invasion, and KRT80 
Table 2 Correlation between KRT80 expression and clinicopathological features

\begin{tabular}{|c|c|c|c|c|}
\hline \multirow[t]{2}{*}{ Parameters } & \multirow{2}{*}{$\begin{array}{l}\text { Total } \\
(n=120)\end{array}$} & \multicolumn{2}{|c|}{ KRT80 protein expression } & \multirow[t]{2}{*}{$P$ value } \\
\hline & & $\begin{array}{l}\text { Negative } \\
(n=55)(\%)\end{array}$ & $\begin{array}{l}\text { Positive } \\
(n=65)(\%)\end{array}$ & \\
\hline \multicolumn{5}{|l|}{ Age, years } \\
\hline$<65$ & 53 & $26(47.3)$ & $27(41.5)$ & \multirow[t]{2}{*}{0.387} \\
\hline$\geq 65$ & 67 & $29(52.7)$ & $38(58.5)$ & \\
\hline \multicolumn{5}{|l|}{ Gender } \\
\hline Male & 67 & $32(58.2)$ & $35(53.8)$ & \multirow[t]{2}{*}{0.577} \\
\hline Female & 53 & 23(41.8) & $30(46.2)$ & \\
\hline \multicolumn{5}{|l|}{ Location } \\
\hline Ascending & 19 & $8(14.5)$ & 11(16.9) & \multirow[t]{5}{*}{0.958} \\
\hline Transverse & 8 & $3(5.5)$ & $5(7.7)$ & \\
\hline Descending & 14 & $7(12.7)$ & $7(10.8)$ & \\
\hline Sigmoid & 43 & $21(38.2)$ & $22(33.8)$ & \\
\hline Rectum & 36 & 16(29.1) & 20(30.8) & \\
\hline \multicolumn{5}{|l|}{ T stage } \\
\hline $\mathrm{T} 1+\mathrm{T} 2$ & 58 & $34(61.8)$ & 24(36.9) & \multirow[t]{2}{*}{$0.013^{\mathrm{a}}$} \\
\hline $\mathrm{T} 3+\mathrm{T} 4$ & 62 & $21(38.2)$ & $41(63.1)$ & \\
\hline \multicolumn{5}{|l|}{ N stage } \\
\hline No & 86 & $47(85.5)$ & $39(60.0)$ & \multirow[t]{2}{*}{$<0.001^{a}$} \\
\hline $\mathrm{N} 1$ & 34 & $8(14.5)$ & $26(40.0)$ & \\
\hline \multicolumn{5}{|l|}{ M stage } \\
\hline MO & 107 & $52(94.5)$ & 55(84.6) & \multirow[t]{2}{*}{$0.004^{\mathrm{a}}$} \\
\hline M1 & 13 & $3(5.5)$ & 10(15.4) & \\
\hline \multicolumn{5}{|l|}{ AJCC stage } \\
\hline$I+\|$ & 59 & $36(65.5)$ & 23(35.4) & \multirow[t]{2}{*}{$0.001^{a}$} \\
\hline$I I I+I V$ & 61 & 19(34.5) & $42(64.6)$ & \\
\hline \multicolumn{5}{|l|}{ Differentiation } \\
\hline Well & 47 & $30(54.5)$ & $17(26.2)$ & \multirow[t]{3}{*}{$0.006^{\mathrm{a}}$} \\
\hline Moderate & 49 & 19(34.6) & $30(46.1)$ & \\
\hline Poor & 24 & $6(10.9)$ & $18(27.7)$ & \\
\hline \multicolumn{5}{|l|}{ Vessel invasion } \\
\hline No & 101 & $51(92.7)$ & 50(76.9) & \multirow[t]{2}{*}{$0.006^{\mathrm{a}}$} \\
\hline Yes & 19 & $4(7.3)$ & 15(23.1) & \\
\hline
\end{tabular}

${ }^{\mathrm{a}} P<0.05$ indicates a significant association among the variables

expression were associated with DFS and OS (Table 3). To further investigate the relationship between the patients' prognosis and individual parameters, multivariate analysis was performed for all significant factors derived from the univariate analysis. The results showed that $\mathrm{M}$ stage, AJCC stage and KRT80 expression were independent prognostic factors for DFS and OS (Table 4).

\section{Construction of stable CRC cells with KRT80 knockdown or overexpression}

First, we checked the expression level of KRT80 in different CRC cells. The results showed that KRT80 was highly expressed in SW620 and Caco-2 cells and lower in RKO cells, as compared with other cells (Fig. 3a, d). To evaluate efficiency of different shRNA sequences and avoid off-target effects, we constructed three plasmids with different shRNA sequences and individually transfected them into SW620 cells. The efficiencies of the shRNAs were confirmed by qRT-PCR (Fig. 4b). Then, shRNA1 and shRNA2 were individually transfected into Caco-2 and SW620 cells. KRT80 plasmid was transfected into RKO cells. Knockdown or overexpression of KRT80 was confirmed by qRT-PCR and western blot (Fig. 3c, e).

KRT80 promotes CRC cell migration and invasion, changes in cell morphology, and EMT markers via the AKT pathway

Cell migration and invasion are necessary for tumor development and metastasis ${ }^{11}$. We performed transwell assay to assess the impact of KRT80 expression on cell migration and invasion. Knockdown of KRT80 suppressed the migration and invasion of CRC cells, whereas overexpression of KRT80 promoted these activities (Fig. 3f, g).

Numerous studies have proven that EMT is necessary for metastasis of malignant cancer ${ }^{12-14}$. Epithelial cells lose their polarity, and acquire migratory and invasive capabilities $^{15}$. The micrographs showed that overexpression of KRT80 in CRC cells can change their morphology from round to polygonal, along with the induction of EMT (Fig. 4b). Immunofluorescent (IF) assay showed lower expression of Vimentin, a mesenchymal marker, after suppression of KRT80, whereas the expression of E-Cadherin, an epithelial marker, was increased (Fig. 4a). Besides, western blot analysis was performed to determine the role of KRT80 in EMT in CRC cells. E-Cadherin was upregulated after KRT80 was suppressed, whereas mesenchymal markers were downregulated, including N-Cadherin, Vimentin, and Snail (Fig. 4c).

The AKT pathway can participate in CRC migration and invasion, so we examined the changes in $\mathrm{AKT}^{16}$. Overexpression of KRT80 promoted the expression of pAKT (Ser 473), whereas the expression of total AKT and p-AKT (Thr 308) showed no significant changes (Fig. 4d).

\section{Suppression of AKT can inhibit EMT expression, cell migration, and invasion}

Afuresertib was reported to be a highly efficient AKT pathway inhibitor ${ }^{17}$. In order to check the function of AKT in CRC, we inhibited AKT expression with 
Table 3 Univariate analysis for disease-free survival (DFS) and overall survival (OS)

\begin{tabular}{|c|c|c|c|c|c|c|}
\hline \multirow[t]{2}{*}{ Variable } & \multicolumn{3}{|l|}{ DFS } & \multicolumn{3}{|l|}{ os } \\
\hline & HR & $95 \% \mathrm{Cl}$ & $P$ value & HR & $95 \% \mathrm{Cl}$ & $P$ value \\
\hline \multicolumn{7}{|l|}{ Age, years } \\
\hline$<65$ & - & & & - & & \\
\hline$\geq 65$ & 0.962 & $0.605-1.530$ & 0.870 & 1.071 & $0.648-1.772$ & 0.788 \\
\hline \multicolumn{7}{|l|}{ Gender } \\
\hline Male & - & & & - & & \\
\hline Female & 0.872 & $0.543-1.399$ & 0.570 & 1.140 & $0.685-1.897$ & 0.614 \\
\hline \multicolumn{7}{|l|}{ Location } \\
\hline Ascending & - & & & - & & \\
\hline Transverse & 1.297 & $0.622-2.705$ & 0.487 & 1.438 & $0.655-3.158$ & 0.365 \\
\hline Descending & 1.978 & $0.786-4.979$ & 0.148 & 2.340 & $0.909-6.022$ & 0.078 \\
\hline Sigmoid & 1.301 & $0.570-2.973$ & 0.532 & 0.730 & $0.244-2.186$ & 0.574 \\
\hline Rectum & 1.112 & $0.627-1.973$ & 0.717 & 1.284 & $0.695-2.372$ & 0.424 \\
\hline \multicolumn{7}{|l|}{ T stage } \\
\hline $\mathrm{T} 1+\mathrm{T} 2$ & - & & & - & & \\
\hline $\mathrm{T} 3+\mathrm{T} 4$ & 2.057 & $1.251-3.381$ & $0.004^{a}$ & 2.200 & $1.281-3.777$ & $0.004^{\mathrm{a}}$ \\
\hline \multicolumn{7}{|l|}{ N stage } \\
\hline No & - & & & - & & \\
\hline $\mathrm{N} 1$ & 2.154 & $1.353-3.429$ & $0.001^{a}$ & 2.474 & $1.497-4.087$ & $<0.001^{a}$ \\
\hline \multicolumn{7}{|l|}{ M stage } \\
\hline MO & - & & & - & & \\
\hline M1 & 3.262 & $1.891-5.629$ & $<0.001^{\mathrm{a}}$ & 4.000 & $2.281-7.014$ & $<0.001^{a}$ \\
\hline \multicolumn{7}{|l|}{ AJCC stage } \\
\hline$I+\|$ & - & & & - & & \\
\hline$I I I+I V$ & 2.451 & $1.519-3.956$ & $<0.001^{a}$ & 2.580 & $1.536-4.334$ & $<0.001^{\mathrm{a}}$ \\
\hline \multicolumn{7}{|l|}{ Differentiation } \\
\hline Well & - & & & - & & \\
\hline Moderate & 2.278 & $1.238-4.192$ & $0.008^{\mathrm{a}}$ & 2.486 & $1.274-4.852$ & $0.008^{\mathrm{a}}$ \\
\hline Poor & 3.560 & $1.865-6.797$ & $<0.001^{a}$ & 3.700 & $1.833-7.468$ & $<0.001^{\mathrm{a}}$ \\
\hline \multicolumn{7}{|c|}{ Vessel invasion } \\
\hline No & - & & & - & & \\
\hline Yes & 2.067 & $1.197-3.568$ & $0.009^{a}$ & 2.166 & $1.208-3.884$ & $0.010^{\mathrm{a}}$ \\
\hline \multicolumn{7}{|c|}{ KRT80 expression } \\
\hline Negative & - & & & - & & \\
\hline Positive & 3.897 & $2.213-6.864$ & $<0.001^{\mathrm{a}}$ & 3.397 & $1.882-6.132$ & $<0.001^{a}$ \\
\hline
\end{tabular}

HR hazard ratio, $\mathrm{Cl}$ confidence interval

${ }^{\mathrm{a}} P<0.05$ was considered significant

Afuresertib. The results showed that suppression of AKT could inhibit the expression of mesenchymal markers, as well as cell migration and invasion, whereas the expression of E-Cadherin was upregulated (Fig. 5). 
Table 4 Multivariate analysis for DFS and OS

\begin{tabular}{|c|c|c|c|c|c|c|}
\hline \multirow[t]{2}{*}{ Variable } & \multicolumn{3}{|l|}{ DFS } & \multicolumn{3}{|l|}{ os } \\
\hline & HR & $95 \% \mathrm{Cl}$ & $P$ value & HR & $95 \% \mathrm{Cl}$ & $P$ value \\
\hline T stage & 1.249 & $0.729-2.140$ & 0.419 & 1.513 & $0.832-2.750$ & 0.175 \\
\hline$N$ stage & 1.184 & $0.709-1.979$ & 0.518 & 1.301 & $0.743-2.279$ & 0.358 \\
\hline M stage & 2.675 & $1.104-6.481$ & $0.029^{a}$ & 4.364 & $1.641-11.603$ & $0.003^{\mathrm{a}}$ \\
\hline AJCC stage & 2.192 & $1.297-3.704$ & $0.003^{a}$ & 2.154 & $1.222-3.796$ & $0.008^{a}$ \\
\hline \multicolumn{7}{|l|}{ Differentiation } \\
\hline Well & - & & & & & \\
\hline Moderate & 1.920 & $0.997-3.698$ & 0.051 & 2.122 & $1.039-4.337$ & $0.039^{a}$ \\
\hline Poor & 2.115 & $1.048-4.267$ & $0.037^{\mathrm{a}}$ & 1.978 & $0.924-4.232$ & 0.079 \\
\hline Vessel invasion & 0.869 & $0.373-2.022$ & 0.744 & 0.609 & $0.232-1.598$ & 0.313 \\
\hline KRT80 expression & 2.527 & $1.390-4.594$ & $0.002^{\mathrm{a}}$ & 2.023 & $1.085-3.771$ & $0.027^{\mathrm{a}}$ \\
\hline
\end{tabular}

$H R$ hazard ratio, $\mathrm{Cl}$ confidence interval

${ }^{\mathrm{a}} P<0.05$ was considered significant

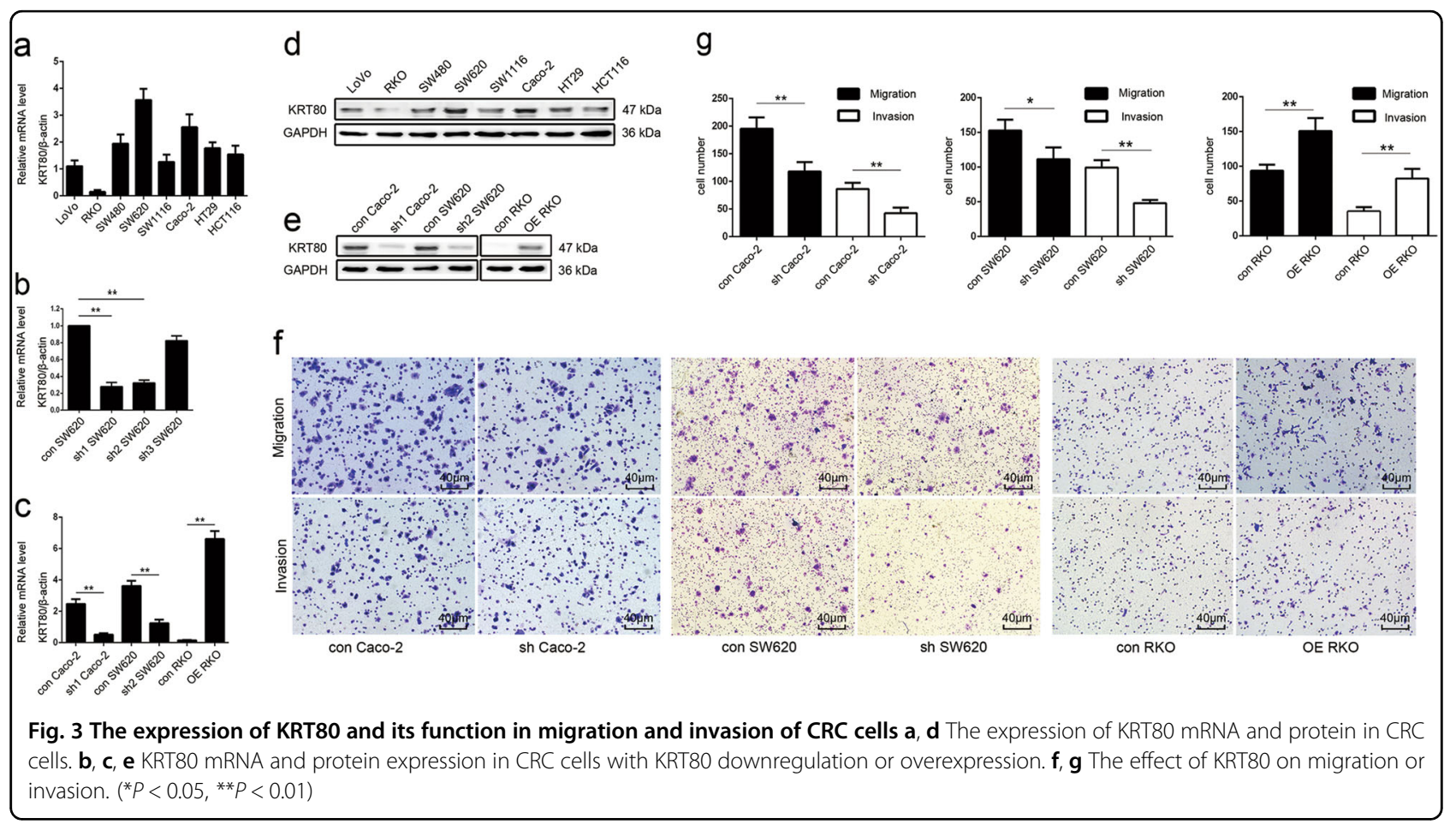

\section{KRT80 interacts with PRKDC in CRC cells}

PRKDC is a member of the phosphatidylinositol-3kinase family ${ }^{18}$. Previous reports have shown that PRKDC is important for AKT activation ${ }^{19,20}$. The results of LCMS/MS showed that KRT80 may interact with PRKDC, so Co-IP assay was performed to test the prediction (Fig. 6a). Next, we used LSCM to assess potential colocalization between KRT80 and PRKDC. The results demonstrated that KRT80 and PRKDC were colocalized mainly around nuclear membranes in CRC cells (Fig. 6b). These data revealed that KRT80 can interact with PRKDC in CRC cells.

\section{Suppression of PRKDC expression can inhibit the} expression of AKT and EMT, cell migration, and invasion

NU7441 can inhibit the expression of PRKDC ${ }^{21}$. Thus, we inhibited PRKDC expression with NU7441, which led to inhibition of the expression of AKT and mesenchymal 


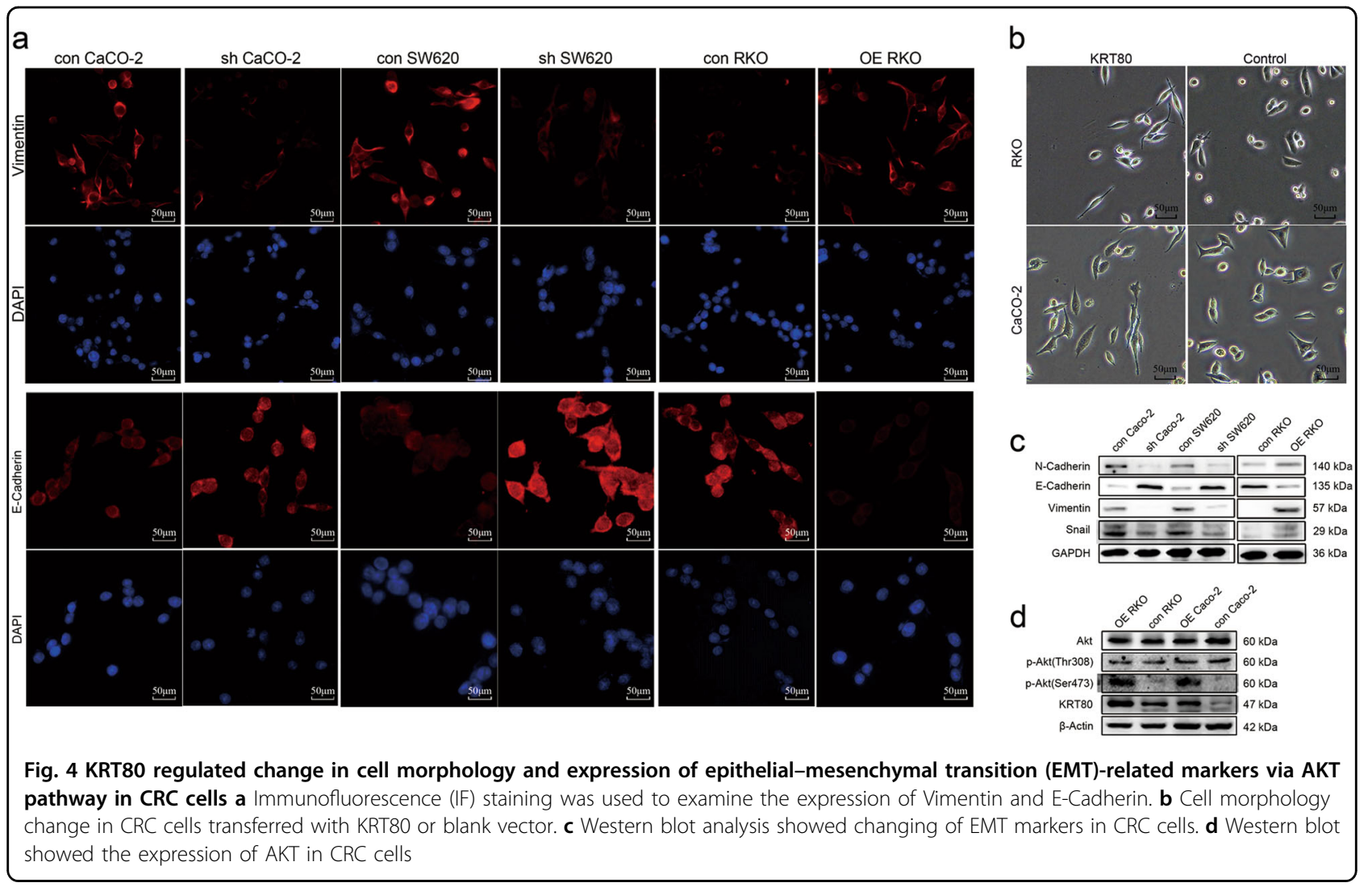

markers as well as CRC cell migration and invasion, whereas the expression of E-Cadherin was upregulated (Fig. 7). Hence, KRT80 can interact with PRKDC, followed by activation of the AKT pathway, which influences cell migration and invasion.

\section{Discussion}

Keratins are markers of epithelial cells ${ }^{22}$. They expressed in normal and malignant cells, and are associated with tumor malignancy and metastasis ${ }^{23}$. Several keratins have been used as prognostic predictors for different carcinomas of epithelial origin ${ }^{24-26}$. Studies have demonstrated that keratins play a vital role in maintaining the mechanical stability and integrity of epithelial cells, and participate in several intracellular signaling pathways involved in cell stress, proliferation, and metastasis ${ }^{27,28}$. Numerous studies have found several genes that were specifically upregulated or downregulated in CRC tissues and could be used as biomarkers for diagnosis or prognosis $^{25,29}$. KRT80 has been rarely reported to be associated with any disease, including cancer.

This study demonstrated that KRT80 was significantly upregulated in CRC, and elevated KRT80 expression correlated with CRC clinicopathological parameters. Patients with KRT80-positive tumor staining had shorter DFS and OS. In addition, Cox regression models showed that KRT80 was an independent prognostic marker for CRC. Furthermore, KRT80 could promote cell migration and invasion. Besides, overexpression of KRT80 could change CRC cell morphology, from round to polygonal. Moreover, the expression of mesenchymal markers were upregulated, whereas epithelial markers were downregulated because of the close relationship between EMT and metastasis of malignant tumors ${ }^{13}$.

Taken together, these results confirmed that KRT80 can promote CRC migration and invasion. Though majority of the previous studies on AKT focused on its function in proliferation, some studies reported its function in metastasis ${ }^{30,31}$. This study was focused on metastasis and revealed that the AKT pathway was strongly associated with CRC migration and invasion since the AKT inhibitor Afuresertib could inhibit these processes.

PRKDC is involved in the ligation step of the nonhomologous end joining pathway of DNA double strand break repair. It also participates in modulation of transcription, maintaining telomere length, regulation of apoptosis, regulation of mitochondrial protein function, and phosphorylation of numerous proteins ${ }^{32-34}$. Also, as a biomarker in malignant tumors, PRKDC participates in tumor metastasis in prostate cancer, laryngeal squamous cell carcinoma and other malignant tumors ${ }^{35-37}$. PRKDC plays numerous functions in tumors, and hence we used 


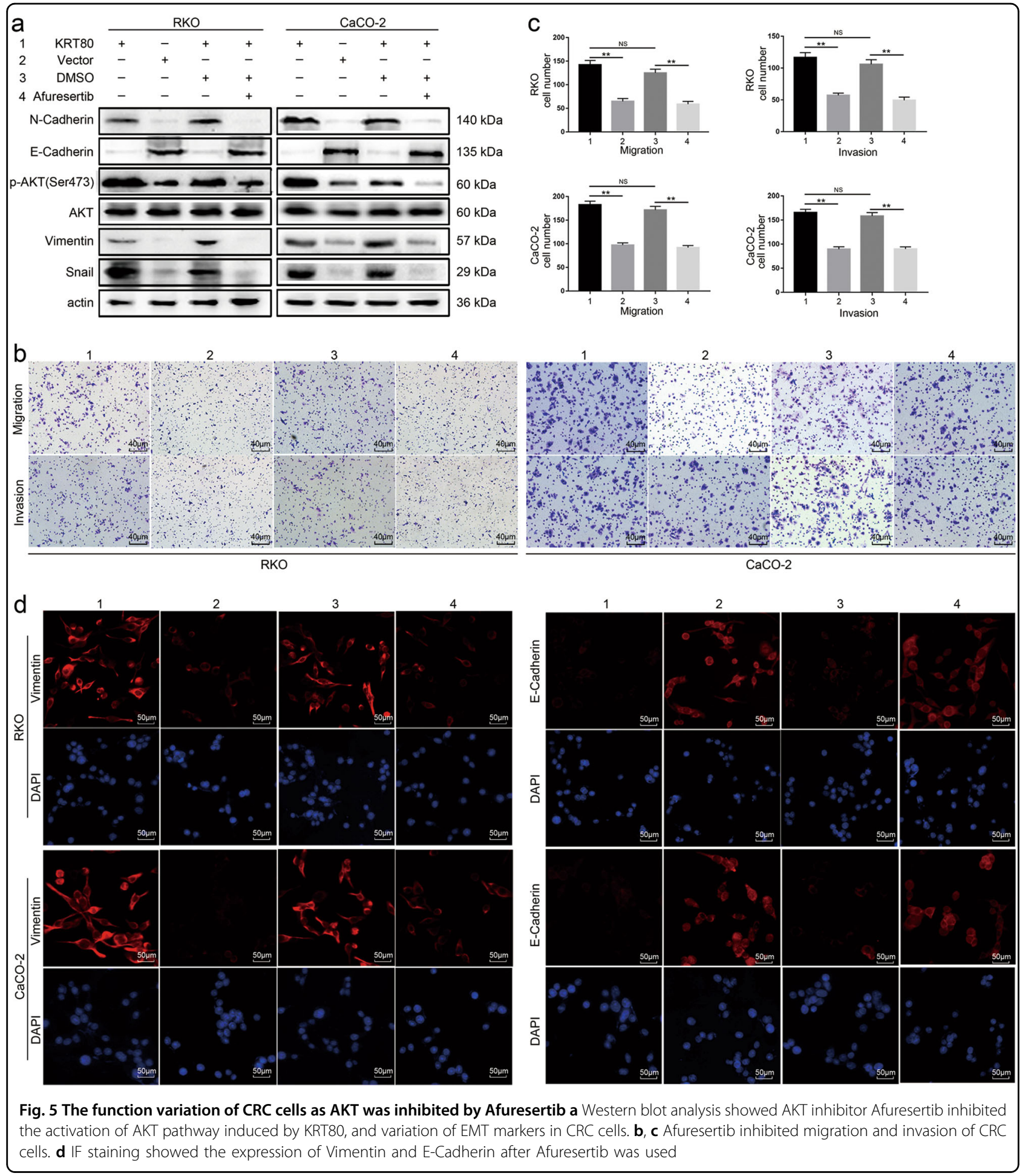

LC-MS/MS to examine its function in this study. The result showed that PRKDC can interact with KRT80, and can influence AKT and cell function.

This is the first report on the function of KRT80 in malignant tumors. KRT80 expression was upregulated in
CRC samples, which significantly correlated with poor survival. These results suggested that KRT80 may be a novel biomarker for the diagnosis of CRC and a therapeutic target for CRC. 

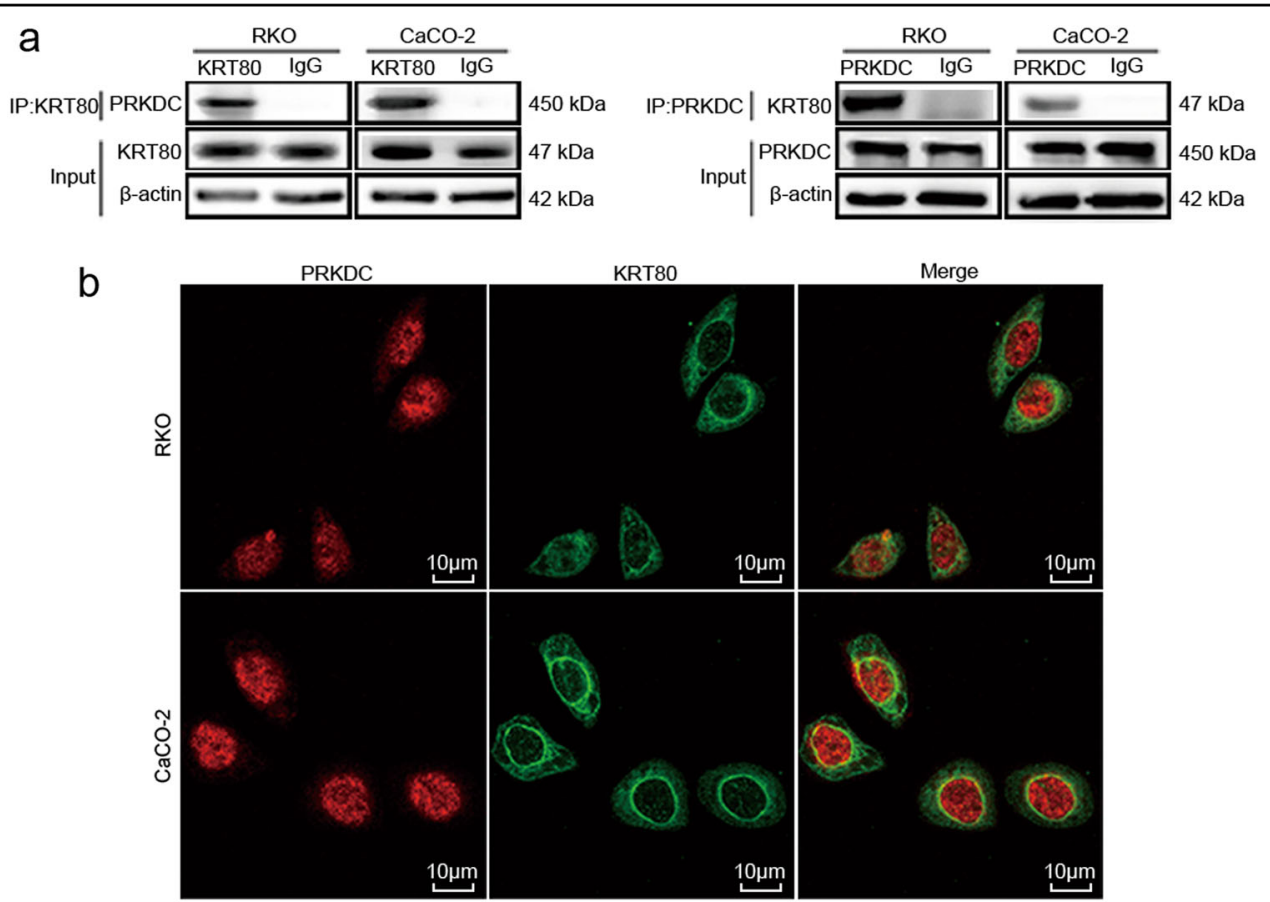

Fig. 6 KRT80 can interact with PRKDC in CRC cells a Co-Immunoprecipitation (CO-IP) assay showed KRT80 can interact with PRKDC in CRC cells. b Laser scanning confocal microscopy (LSCM) technique was used to show the colocalization of KRT80 and PRKDC in CRC cells

\section{Materials and methods \\ Databases analysis}

With the approval of the project by the consortium, TCGA RNA-Seq were downloaded from TCGA website (https://cancergenome.nih.gov/). Oncomine (https:// www.oncomine.org/) was used to acquire KRT80 RNA expression in CRC and normal mucosae.

\section{Patients and tissue samples}

One hundred and twenty CRC tissue samples for TMA construction and other 40 CRC tissues were obtained from the Department of General Surgery, Shanghai General Hospital. Patients who had received chemotherapy or radiotherapy were excluded from this study. DFS and OS was defined as the interval between the initial surgery to clinical or radiological determined recurrence/ metastasis or death. Tumor staging was based on pathological outcomes based on the guidelines of AJCC. The diagnoses were confirmed by at least two clinical pathologists. Written informed consents were obtained from all patients. The study was approved by the Ethics Committee of Shanghai General Hospital.

\section{QRT-PCR}

Total RNA was isolated from 40 pairs of CRC tissues and cell lines, then was used to synthesize cDNA (TaKaRa, Tokyo, Japan), according to the manufacturer's instructions. KRT80 mRNA levels were quantitated using SYBR Green Master Mix Kit (Takara), qRT-PCR was performed using the Real-time PCR System (Applied Biosystems, Foster City, CA, USA). The amplification conditions used were as follows: initial denaturation for $30 \mathrm{~s}$ at $95^{\circ} \mathrm{C}, 40$ cycles of denaturation for $5 \mathrm{~s}$ at $95^{\circ} \mathrm{C}$, annealing for $30 \mathrm{~s}$ at $60^{\circ} \mathrm{C}$, and elongation for $30 \mathrm{~s}$ at $72{ }^{\circ} \mathrm{C}$, with a final extension step for $30 \mathrm{~s}$ at $72^{\circ} \mathrm{C}$. RT-qPCR was performed in triplicate and the fold-change $\left(2^{-\Delta \Delta C t}\right)$ of KRT80 expression was calculated for each group. The primers used for quantitative PCR are:

KRT80 forward: 5' - GCTGCTCTTGCCATAATCAA-3'

KRT80 reverse: 5'-AATGCTCCTGCCCAATCTC-3'

$\beta$-actin forward: $5^{\prime}$-CATGTACGTTGCTATCCAGGC-3' $\beta$-actin reverse: $5^{\prime}$-CTCCTTAATGTCACGCACGAT-3'

\section{Protein extraction and western blot}

Total protein from CRC tissue samples and cells were extracted using radioimmunoprecipitation assay buffer (RIPA) Lysis Buffer (Beyotime Biotechnology Co., Jiangsu, China). Western blot was performed as previously described ${ }^{38}$. The following primary antibodies were used: anti-KRT80 (1:1000, Proteintech Group, Inc., Rosemont, IL, USA), anti- N-Cadherin (1:1000, Cell Signaling Technology, CST, Danvers, MA, USA), anti-E-Cadherin (1:1000, CST), anti-Vimentin (1:1000, CST), anti-Snail (1:1000, CST), anti-AKT (1:1000, CST), anti-p-AKT (Thr 

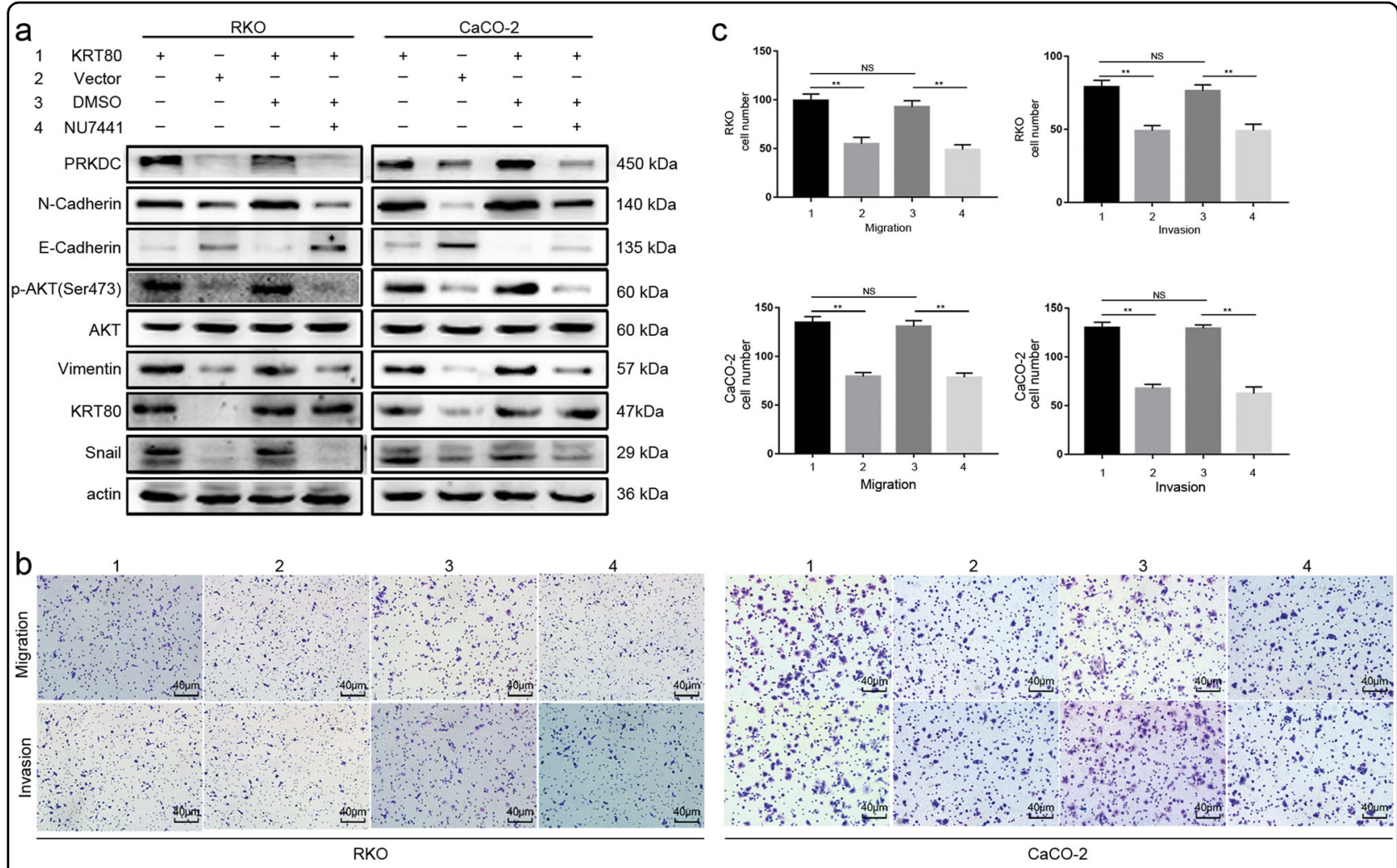

d
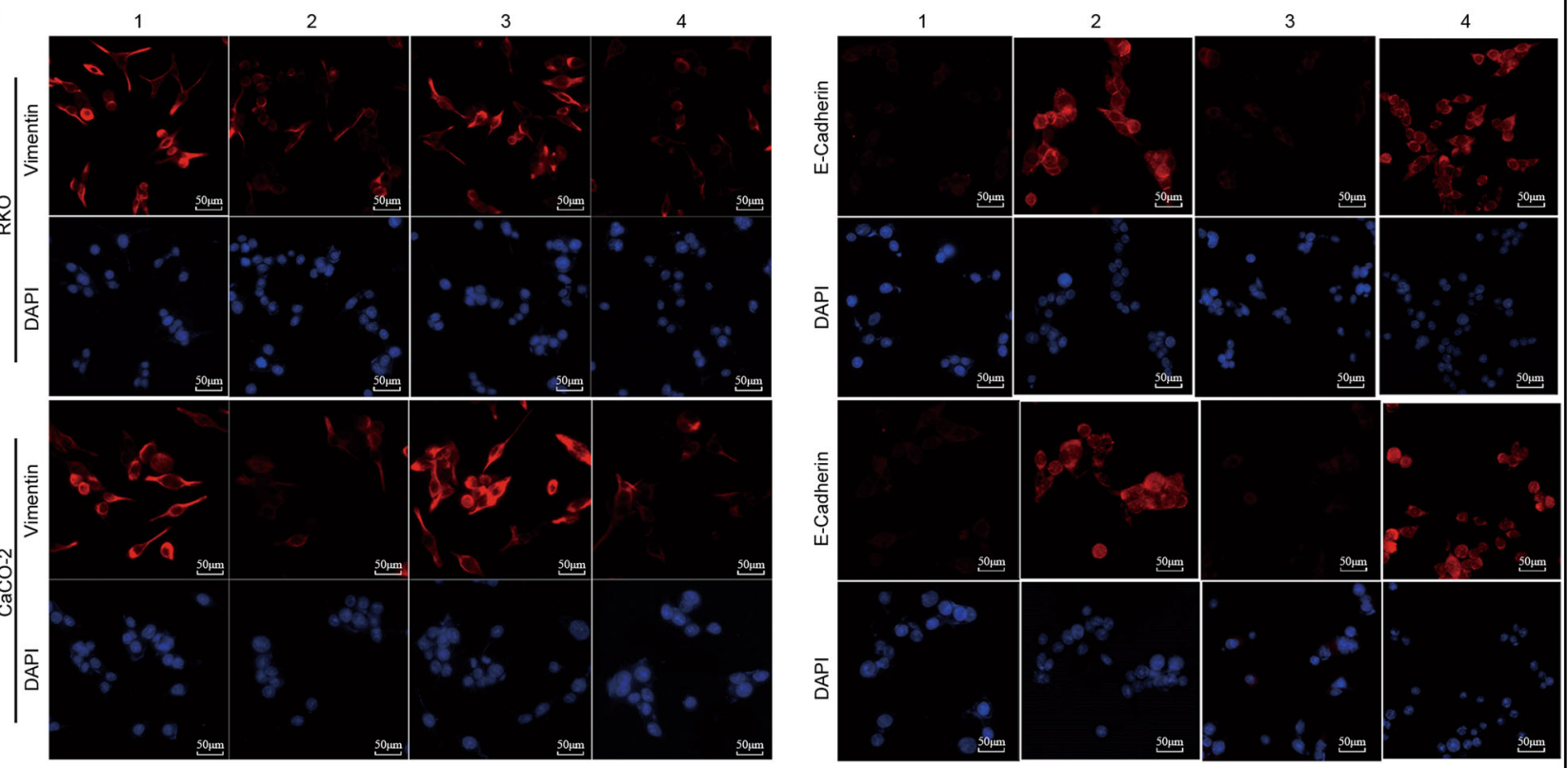

Fig. 7 Inhibit PRKDC expression with NU7441 in CRC cells a Western blot analysis showed PRKDC inhibitor NU7441 inhibited the expression of PRKDC, which further inhibited the activation of AKT pathway induced by KRT80 and EMT markers in CRC cells. b, $\mathbf{c}$ The suppression of PRKDC with NU7441 inhibited migration and invasion of CRC cells. $\mathbf{d}$ IF staining showed the expression of Vimentin and E-Cadherin were changed as NU7441 inhibited PRKDC expression

308) (1:1000, CST), anti-p-AKT (Ser 473) (1:1000, CST), anti-PRKDC (1:1000, CST), anti-GAPDH (1:1000, CST), anti- $\beta$-Actin $(1: 1000$, CST).

\section{IHC}

TMA that included $120 \mathrm{CRC}$ tissues were constructed by commercial company (Outdo Biotech Co. Shanghai, 
China). IHC staining of TMA and the calculating method were described before ${ }^{39}$.

\section{Plasmid transfection}

KRT80 shRNA was constructed by Genechem Co. Ltd. (Shanghai, China) and was used to silence KRT80. Genechem (Shanghai) also synthesized the KRT80 plasmid. The KRT80 shRNA and KRT80 primer sequences are listed below:

shRNA1 forward: GCACTATCTCCAAGGTGACTGT GAA

shRNA1 reverse: TTCACAGTCACCTTGGAGATAG TGC

shRNA2 forward: GGATGCAGAGTGTCTTCATCG shRNA2 reverse: CGATGAAGACACTCTGCATCC shRNA3 forward: GCCATTGCCTGAAACTGGAGGA

GAA

shRNA3 reverse: TTCTCCTCCAGTTTCAGGCAAT GGC

KRT80 forward: ATGGCCTGCCGCTCCTGCGTGGTT KRT80 reverse: TTACTCTGAGACCTCCGACTCCT

CRC cells were infected with $5 \times 10^{5}$ transducing units/ $\mathrm{ml}$ of lentiviral particles. Stable cell lines were established after antibiotic selection using $1 \mu \mathrm{g} / \mathrm{ml}$ puromycin (Sigma, St. Louis, MO, USA). KRT80 expression was confirmed in CRC cells using qRT-PCR and western blot analysis.

\section{Migration and invasion assays}

In total, $1 \times 10^{5}$ cells cultured in fetal bovine serum (FBS)-free media were seeded onto the upper chambers of transwell (Corning, NY, USA), and media with $10 \%$ FBS was added to the lower chamber. Upper chamber coated without Matrigel (Coring) for migration assay or with Matrigel for invasion assay. After incubating for appropriate intervals, chamber was fixed in methanol and then stained using crystal violet (Beyotime). Using a light microscope, at least five randomly selected fields were photographed following which the counts were averaged. All experiments were performed in triplicate.

\section{IF staining}

Cells were seeded into six well plate with $10 \mathrm{~mm}$ coverslip for $24 \mathrm{~h}$. After washing with phosphate-buffered saline, cells were fixed in $4 \%$ paraformaldehyde. Then incubated with primary antibody overnight at $4{ }^{\circ} \mathrm{C}$. After incubating the specimen with fluorochrome-conjugted secondary antibody and 4',6-diamidino-2-phenylindole lucifugally. Images were obtained with a fluorescence microscope.

\section{Co-IP assays}

The cells were incubated in weak-potency RIPA lysis buffer (Beyotime) for $30 \mathrm{~min}$ at $4{ }^{\circ} \mathrm{C}$, then centrifuged at $12000 \mathrm{~g}$ for $30 \mathrm{~min}$. Antibodies against PRKDC and
KRT80 were added with protein $\mathrm{A}$ and $\mathrm{G}$ agarose beads (Sigma), and incubated at $4{ }^{\circ} \mathrm{C}$ for $6 \mathrm{~h}$. After washing, the complexes were boiled and subjected to western blotting analysis.

\section{Statistical analysis}

All data were presented as mean \pm SD for continuous variables or frequencies and percentages for categorical data. Comparisons between the two groups were performed using the students' $t$ test and three-group comparisons were performed using one-way analysis of variance. The $\chi^{2}$ or Fisher's exact tests was used to determine the significance of difference between KRT80 and clinicopathological variables. Kaplan-Meier analyses with log-rank test were used to evaluate DFS and OS. The Cox proportional hazard model was used to investigate the hazard ratio and 95\% confidence intervals for DFS and OS. All data analysis was performed using the SPSS 20.0 software (SPSS Inc, Chicago, IL, USA). $P$ value $<0.05$ was considered statistically significant.

\section{Acknowledgements}

The work was supported by Natural Science Foundation of China (NSFC)-81773188, 81760557, 81703081 and Program for New Century Excellent Talents in University (NCET)-12-0381. And we would like to appreciate the help given to the work by the lab of Prof. Fuyu Yang's from institute of Biophysics and Prof. Yaping Tu from Creighton University in USA.

\section{Author details \\ ${ }^{1}$ Department of General Surgery, Tongji Hospital, Tongji University School of Medicine, Shanghai 200065, China. ${ }^{2}$ Department of General Surgery, Shanghai General Hospital, Shanghai Jiao Tong University School of Medicine, Shanghai 200080, China. ${ }^{3}$ Department of General Surgery, Zhejiang Hospital, Hangzhou 310013, China. ${ }^{4}$ Department of General Surgery, The Second Affiliated Hospital of Jiaxing College, Jiaxing 314000, China. ${ }^{5}$ Shanghai Institute of Immunology, Shanghai Jiao Tong University School of Medicine, Shanghai 200025, China}

Conflict of interest

The authors declare that they have no conflict of interest.

\section{Publisher's note}

Springer Nature remains neutral with regard to jurisdictional claims in published maps and institutional affiliations.

Received: 10 February 2018 Revised: 30 August 2018 Accepted: 4 September 2018

Published online: 27 September 2018

\footnotetext{
References

1. Mercier, J. \& Voutsadakis, I. A. A systematic review and meta-analysis of retrospective series of regorafenib for treatment of metastatic colorectal canacer. Anticancer Res. 37, 5925-5934 (2017).

2. Group, A. W., Ccm \& Group, A. W. Italian cancer figures, report 2012: cancer in children and adolescents. Epidemiol. Prev. 37(1Suppl 1), 1-225 (2013).

3. Ugras, N., Ozgun, G., Ocakoglu, G., Yerci, O. \& Ozturk, E. Relationship between HER-2, COX-2, p53 and clinicopathologic features in gastric adenocarcinoma. Do these biomarkers have any prognostic significance? Turk. J. Gastroenterol. 25, 176-181 (2014).

4. Uenishi, T. et al. Cytokeratin 19 expression in hepatocellular carcinoma predicts early postoperative recurrence. Cancer Sci. 94, 851-857 (2003).
} 
5. Strnad, P., Paschke, S., Jang, K. H. \& Ku, N. O. Keratins: markers and modulators of liver disease. Curr. Opin. Gastroenterol. 28, 209-216 (2012).

6. Rogers, M. A. et al. Characterization of new members of the human type ॥ keratin gene family and a general evaluation of the keratin gene domain on chromosome 12q13.13. J. Invest. Dermatol. 124, 536-544 (2005).

7. Omary, M. B., Ku, N. O., Strnad, P. \& Hanada, S. Toward unraveling the complexity of simple epithelial keratins in human disease. J. Clin. Invest. 119, 1794-1805 (2009).

8. Kurokawa, I. et al. Keratin profiles may differ between intraepidermal and intradermal invasive eccrine porocarcinoma. Oncol. Rep. 16, 473-477 (2006).

9. Wu, P. C. et al. Classification of hepatocellular carcinoma according to hepatocellular and biliary differentiation markers. Clinical and biological implications. Am. J. Pathol. 149, 1167-1175 (1996).

10. Govaere, O. et al. Keratin 19: a key role player in the invasion of human hepatocellular carcinomas. Gut 63, 674-685 (2014).

11. Cui, Y. M. et al. FOXC2 promotes colorectal cancer metastasis by directly targeting MET. Oncogene 34, 4379-4390 (2015).

12. Li, C. et al. GDF15 promotes EMT and metastasis in colorectal cancer. Oncotarget 7, 860-872 (2016).

13. Cai, L. M. et al. EBV-miR-BART7-3p promotes the EMT and metastasis of nasopharyngeal carcinoma cells by suppressing the tumor suppressor PTEN. Oncogene 34, 2156-2166 (2015).

14. Parvani, J. G., Gujrati, M. D., Mack, M. A., Schiemann, W. P. \& Lu, Z. R. Silencing beta3 integrin by targeted ECO/siRNA nanoparticles inhibits EMT and metastasis of triple-negative breast cancer. Cancer Res. 75, 2316-2325 (2015).

15. Elsum, I. A., Martin, C. \& Humbert, P. O. Scribble regulates an EMT polarity pathway through modulation of MAPK-ERK signaling to mediate junction formation. J. Cell Sci. 126, 3990-3999 (2013).

16. Rui, X., Yan, X. I. \& Zhang, K. Baicalein inhibits the migration and invasion of colorectal cancer cells via suppression of the AKT signaling pathway. Oncol. Lett. 11, 685-688 (2016).

17. Spencer, A. et al. The novel AKT inhibitor afuresertib shows favorable safety, pharmacokinetics, and clinical activity in multiple myeloma. Blood 124, 2190-2195 (2014).

18. Mannell, H., Hammitzsch, A., Mettler, R., Pohl, U. \& Krotz, F. Suppression of DNAPKcs enhances FGF-2 dependent human endothelial cell proliferation via negative regulation of Akt. Cell Signal. 22, 88-96 (2010).

19. $\mathrm{Hu}, \mathrm{H}$. et al. DNA-PKcs is important for Akt activation and gemcitabine resistance in PANC-1 pancreatic cancer cells. Biochem. Biophys. Res. Commun. 452, 106-111 (2014).

20. Fang, Y. et al. DNA-PKcs deficiency sensitizes the human hepatoma HepG2 cells to cisplatin and 5-fluorouracil through suppression of the PI3K/Akt/NFkappaB pathway. Mol. Cell Biochem. 399, 269-278 (2015).

21. Ciszewski, W. M., Tavecchio, M., Dastych, J. \& Curtin, N. J. DNA-PK inhibition by NU7441 sensitizes breast cancer cells to ionizing radiation and doxorubicin. Breast Cancer Res. Treat. 143, 47-55 (2014).

22. Fortier, A. M., Asselin, E. \& Cadrin, M. Keratin 8 and 18 loss in epithelial cancer cells increases collective cell migration and cisplatin sensitivity through claudin1 up-regulation. J. Biol. Chem. 288, 11555-11571 (2013).
23. Wang, $X$. et al. Oblongifolin $C$ inhibits metastasis by up-regulating keratin 18 and tubulins. Sci. Rep. 5, 10293 (2015).

24. Xiang, Z. L. et al. Expression of cytokeratin 19 and matrix metalloproteinase 2 predicts lymph node metastasis in hepatocellular carcinoma. Mol. Biol. Rep. 38, 3531-3539 (2011).

25. Czapiewski, P. et al. Keratin 7 expression in lymph node metastases but not in the primary tumour correlates with distant metastases and poor prognosis in colon carcinoma. Pol. J. Pathol. 67, 228-234 (2016).

26. Polioudaki, H. et al. Variable expression levels of keratin and vimentin reveal differential EMT status of circulating tumor cells and correlation with clinical characteristics and outcome of patients with metastatic breast cancer. BMC Cancer 15, 399 (2015).

27. Zhang, Q. et al. Mechanical and biological properties of oxidized horn keratin. Mater. Sci. Eng. C. Mater. Biol. Appl. 47, 123-134 (2015).

28. Tang, J. et al. A novel biomarker Linc00974 interacting with KRT19 promotes proliferation and metastasis in hepatocellular carcinoma. Cell Death Dis. 5, e1549 (2014).

29. Misiorek, J. O. et al. Keratin 8-deletion induced colitis predisposes to murine colorectal cancer enforced by the inflammasome and IL-22 pathway. Carcinogenesis 37, 777-786 (2016).

30. Kayastha, F., Madhu, H., Vasavada, A. \& Johar, K. Andrographolide reduces proliferation and migration of lens epithelial cells by modulating PI3K/Akt pathway. Exp. Eye Res. 128, 23-26 (2014).

31. Wang, $H$. et al. Activation of phosphatidylinositol 3-kinase/Akt signaling mediates sorafenib-induced invasion and metastasis in hepatocellular carcinoma. Oncol. Rep. 32, 1465-1472 (2014).

32. Um, J. H. et al. Involvement of DNA-dependent protein kinase in regulation of the mitochondrial heat shock proteins. Leuk. Res. 27, 509-516 (2003).

33. Dip, R. \& Naegeli, H. More than just strand breaks: the recognition of structural DNA discontinuities by DNA-dependent protein kinase catalytic subunit. FASEB J. 19, 704-715 (2005).

34. Gustafsson, A. S., Abramenkovs, A. \& Stenerlow, B. Suppression of DNAdependent protein kinase sensitize cells to radiation without affecting DSB repair. Mutat. Res. 769, 1-10 (2014).

35. Goodwin, J. F. et al. DNA-PKcs-mediated transcriptional regulation drives prostate cancer progression and metastasis. Cancer Cell. 28, 97-113 (2015).

36. Kang, G. Y. et al. Inhibition of Snail1-DNA-PKcs protein-protein interface sensitizes cancer cells and inhibits tumor metastasis. J. Biol. Chem. 288, 32506-32516 (2013)

37. He, S. S. et al. DNA-dependent protein kinase catalytic subunit functions in metastasis and influences survival in advanced-stage laryngeal squamous cell carcinoma. J. Cancer 8, 2410-2416 (2017).

38. Wang, Z. et al. STYK1 promotes epithelial-mesenchymal transition and tumor metastasis in human hepatocellular carcinoma through MEK/ERK and PI3K AKT signaling. Sci. Rep. 6, 33205 (2016).

39. Li, C. et al. Overexpression of DBC1, correlated with poor prognosis, is a potential therapeutic target for hepatocellular carcinoma. Biochem. Biophys. Res. Commun. 494, 511-517 (2017). 